\title{
The influence of postoperative coronal alignment on revision surgery in total knee arthroplasty
}

\author{
Samer S. Morgan • A. Bonshahi • N. Pradhan • \\ A. Gregory • A. Gambhir • M. L. Porter
}

Received: 16 April 2007 / Accepted: 28 April 2007 / Published online: 5 July 2007

(C) Springer-Verlag 2007

\begin{abstract}
This study examines the association between postoperative coronal tibiofemoral alignment and revision surgery in knee arthroplasty. We retrospectively reviewed the case notes and post-operative long-leg radiographs of 197 Kinemax knee arthroplasty with mean follow-up of 9 years (SD 2.2). They were divided into three groups: neutral, valgus and varus. Revision or decision to revise was used as a hard endpoint. There was no statistical difference among the three groups $(\mathrm{p}=0.78)$. We conclude that aseptic failure of a total knee is multifactorial. Coronal tibio-femoral alignment may not be as important a cause of failure as has been previously thought.
\end{abstract}

Résumé Cette étude a pour but d'examiner l'alignement des éléments prothétiques dans la chirurgie de reprise de prothèse totale du genou. Nous avons de façon rétrospective revu en 1997 une série de prothèses de type Kinemax avec un suivi moyen de 9 ans (SD 2.2). La population a été divisée en trois groupes : Neutre, Valgus et Varus. Pour les courbes de survie ont été utilisées comme référence la révision ou la décision de révision. Nous pouvons conclure que le descellement aseptique d'une prothèse totale du genou est multi factorielle. L'alignement tibio fémoral n'est pas une cause importante d'échec comme l'on pouvait le penser auparavant.

S. S. Morgan $(\bowtie)$

38 Durham Street, Wigan,

WN1 3YN Greater Manchester, UK

e-mail: samermorgan@yahoo.com

S. S. Morgan · A. Bonshahi · N. Pradhan - A. Gregory •

A. Gambhir · M. L. Porter

Wrightington Hospital,

Hall Lane, Appley Bridge, Wigan,

Greater Manchester WN6 9EP, UK

\section{Introduction}

The predictive value of post-operative coronal tibiofemoral alignment on the outcome of total knee replacement is a contentious issue. The orthopaedic literature contains numerous reports recommending that reproduction of the coronal tibiofibular angle close to anatomical valgus is likely to achieve improved outcome $[2,4,5,8,9]$. The rationale behind this hypothesis is that the inability to achieve perfect alignment would directly contribute to early failure.

Jeffery et al. [7] reported that a deviation of alignment of more than 3 degrees from the mechanical axis significantly increased the incidence of loosening of the Denham knee replacement. Conversely Smith et al. [11] reporting a series of Insall-Burnstein knee arthroplasties were unable to confirm the importance of alignment in the long-term survival of the prosthesis. The association between coronal alignment and revision surgery has not been previously studied using long-leg films.

In this study we retrospectively reviewed 197 knees using long-leg radiographs to examine the association between post-operative coronal alignment in total knee arthroplasty and revision sugery.

\section{Methods and materials}

In our institution all patients undergoing total knee replacement routinely undergo both pre- and post-operative long-leg films, showing the hip, knee and ankle all on one radiograph. Patients are routinely followed up at annual or biannual intervals indefinitely. This involves both clinical and radiological review. Accordingly we retrospectively reviewed long-leg radiographs and case notes of 153 
patients who underwent 197 posterior cruciate retaining primary total knee replacements, performed between 1990 and 1993 under the care of a single consultant orthopaedic surgeon with a mean follow-up of 9 years (SD 2.2). The date of the last follow-up was determined by the last entry in the clinic notes.

One hundred and nine knees were operated on in female patients and 44 in male patients. The indications for surgery were osteoarthritis in 128 knees and rheumatoid arthritis in 69 knees. Mean age at operation was 64.9 (SD 8.9). All patients had postoperative long-leg anteroposterior plain radiographs from which coronal tibiofemoral alignment was measured. We performed measurements on the immediate post-operative films. Standard radiographic technique was applied to all using either $105 \times 35$ or $90 \times 30 \mathrm{~cm}$ films with the distance from tube set to 260 or $200 \mathrm{~cm}$, respectively. The tibio-femoral angle for each patient was measured. The angle is found by intersecting the femoral anatomical axis with the tibial anatomical axis. The 197 knees were classified into three groups according to the tibiofemoral angle:

1. Neutral group - 4 to 9 of anatomical valgus;

2. Valgus group - from 9.1 degrees and above;

3. Varus group - from 3.9 degree and below.

\section{Statistical analysis}

Revision or decision to revise was used as a hard endpoint in this study. Statistical analyses were performed using the statistical software package SPSS version 13.0. First the data were subjected to descriptive analysis. The survival among the three groups was constructed using the Kaplan Meier survival curve (Fig. 2).

Table 1 Characteristics of the three alignment groups

\begin{tabular}{lccc}
\hline Alignment & Neutral & Valgus & Varus \\
\hline Mean (SD) age at operation & 64.6 & 64.9 & 65.4 \\
& $(9.4)$ & $(8.8)$ & $(8.4)$ \\
Pathology & & & \\
Osteoarthritis (\%) & 47 & 30 & 51 \\
& $(64.4)$ & $(51.7)$ & $(77.7)$ \\
Rheumatoid arthritis(\%) & 26 & 28 & 15 \\
& $(35.6)$ & $(48.3)$ & $(22.7)$ \\
Mean follow-up in years (SD) & 9.3 & 8.6 & 9.3 \\
& $(2.0)$ & $(2.7)$ & $(2.0)$ \\
Mean(SD) BMI & 25.7 & 25.6 & 27.3 \\
& $(4.5)$ & $(5.4)$ & $(2.3)$ \\
Mean(SD) coronal tibio-femoral & 6.6 & 12.4 & 1.7 \\
angle & $(1.3)$ & $(3.3)$ & $(2.7)$ \\
\hline
\end{tabular}

Values are numbers (percentages) unless stated otherwise
Table 2 Number and median time of death post-operatively in the three alignment groups

\begin{tabular}{llll}
\hline Alignment group & Neutral & Valgus & Varus \\
\hline No. of deaths post-operatively & 12 & 9 & 6 \\
Median time of death & 9.1 & 6.8 & 8.1 \\
$\quad$ postoperatively & $(8-11)$ & $(5-8)$ & $(6-10)$ \\
\hline
\end{tabular}

Survival times were censored at 11 years as beyond that time follow-up appeared to be related to survival, which would result in biased survival estimates. Log rank test was used to show any significant difference in survival between the three groups.

\section{Results}

A total of 153 patients (197 knees) aged 35 to 84 years were involved in the study. Those knees were divided into three alignment groups, the neutral group $(n=73)$, valgus group $(n=58)$ and varus group $(n=66)$. Table 1 shows that the three groups are comparable in terms of age, follow-up, pathology and BMI.

Table 2 shows the number and median time of death post-operatively in the three alignment groups.

A total of six knees were revised because of aseptic loosening or instability. In our cohort there were no revisions because of infection. The distribution and cause of revisions among the three groups are shown in Table 3. Figure 1 shows the value of the coronal tibio-femoral angle in the three alignment groups.

Revision or decision to revise was used as a hard endpoint. The survival among the three alignment groups was demonstrated using Kaplan Meier survival curve (Fig. 2). Log rank test showed a p-value of 0.78 .

\section{Discussion}

Many authors highlight the importance of accurate coronal alignment in influencing the outcomes of total knee replacement $[1,6,7]$. However, our results showed no

Table 3 Number and cause of revisions in the three alignment groups

\begin{tabular}{llll}
\hline Alignment & $\begin{array}{l}\text { Neutral } \\
\mathrm{n}=73\end{array}$ & $\begin{array}{l}\text { Valgus } \\
\mathrm{n}=58\end{array}$ & $\begin{array}{l}\text { Varus } \\
\mathrm{n}=66\end{array}$ \\
\hline $\begin{array}{l}\text { Cause of revision } \\
\text { Instability }\end{array}$ & 1 & 0 & 1 \\
Aseptic loosening & 2 & 1 & 1 \\
Total & 3 & 1 & 2 \\
\hline
\end{tabular}




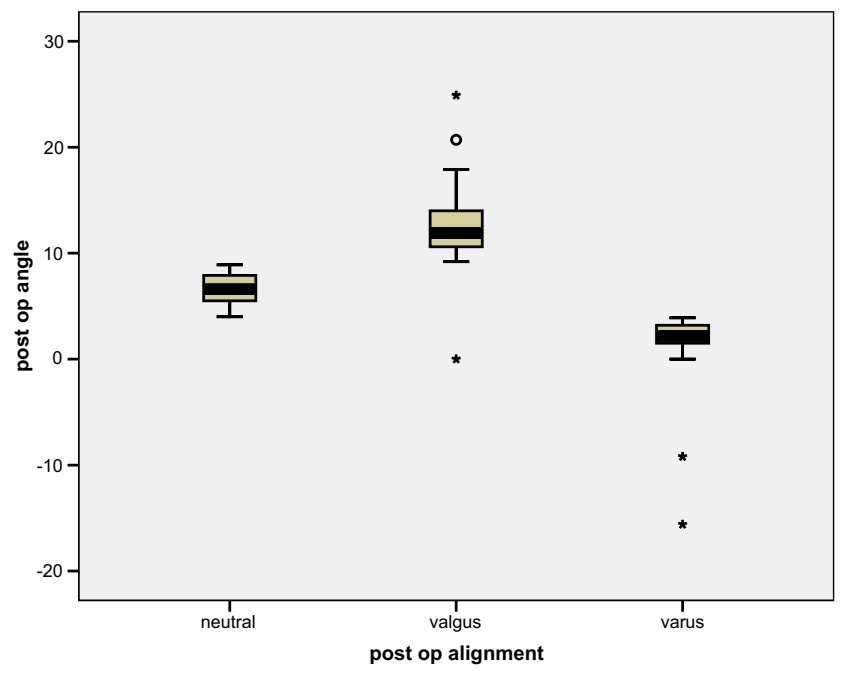

Fig. 1 Box plot showing value of post-operative coronal angle in the three groups

association between immediate post-operative coronal alignment and revision surgery $(\mathrm{p}=0.8)$. This leads to an important question: what is the correct alignment? The limits of such correct alignment have not been established from a strong evidence base nor has the specific correct alignment for each individual knee.

Insall [6] predicts an unsatisfactory result if the alignment is not correct to within 5 degrees of the accepted range. He did not present analysed data to support this assertion. Ritter et al. [10] studied 421 posterior cruciate condylar total knee arthroplasties. In this study there were

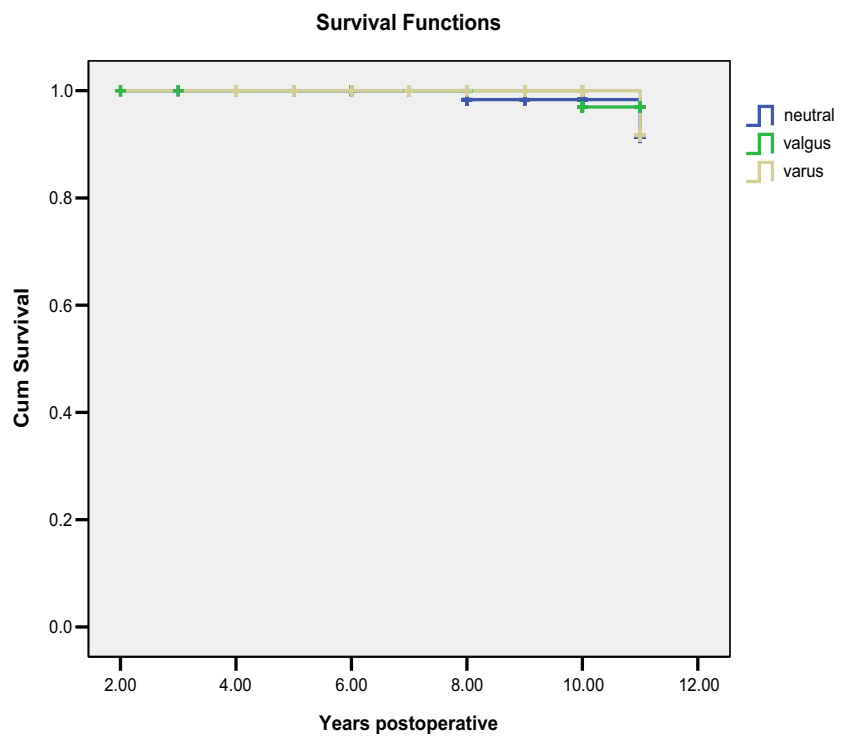

Fig. 2 Kaplan Meier survival curve showing the survival among the three groups. Log rank test showed no significant difference among the three alignment groups; $p$ value 0.78 eight failures, five in the varus group, three in the neutral group and none in the valgus group. He concluded that the surgeons should strive to align the prosthesis in neutral or just a slight amount of anatomical valgus alignment. In his study Ritter reported only the total number of revisions in each group without stating the reason for revision, which could possibly bias the results if the revisions were for other causes not linked to malalignment, e.g., infection.

On the other hand Tew and Waugh [12] showed that malalignment was not the most important cause of failure in the knees that they studied. They observed the relationship between alignment and outcome in 428 knees using shortleg films and concluded that "incorrect" or "abnormal" alignment is not always associated with failure and, conversely, that failure is not always associated with malalignment.

The relationship between alignment and radiolucencies is not significant as shown by Harvey et al. [3], who reviewed 101 knees with cemented tibial components with a minimum follow-up of 5 years to establish whether errors of alignment have a significant effect on the rate of radiological loosening of the prosthesis. Using two methods of assessing radiolucencies, there was a non-significant relationship between alignment and radiolucencies. Similarly Smith et al. [11] reviewed 65 Insall-Burnstein arthroplasties with a mean follow-up of approximately 4 years and found no relation between alignment and progressive radiolucent lines.

In our study we chose revision or decision to revise as the hard endpoint and did not determine and measure the radiolucent lines as it is a somewhat subjective process. Moreover these lines are affected by small changes in the projection angle.

With long-leg radiographs, there should be no difference in using either the mechanical or coronal axis. We used coronal alignment rather than the mechanical axis because it is more commonly used in the published work and allows for comparison.

Previous studies used short-leg films in assessing alignment, which can lead to inaccuracies when measuring the tibio-femoral alignment. In our study each patient had standing full-length post-operative radiographs taken in a standardised manner. This method has been shown to be more accurate than short-leg films in determining tibiofemoral alignment [13]. Peterson et al. [13] studied the anteroposterior radiographs of 50 knees to compare the tibiofemoral alignment as measured on short-leg films with that measured on long-leg films. The mean difference of the tibiofemoral angle measurements between short and long films was $1 \cdot 4^{0} \pm 2 \mathrm{SD}=-3$ to +5 . This was statistically significant $(\mathrm{P}<0.001)$.

Of the previous studies that investigated the relations between alignment and survival, our study has the longest follow-up (mean follow-up of 9 years). 
Our study used revision as the endpoint, but there may be other causes of failure in a knee replacement such as pain, asymptomatic loosening, etc., for which a revision has not been performed or planned. These factors, though important, fall beyond the purview of our study, which aims to evaluate whether there is an association between postoperative coronal alignment and revision surgery. Also we have analysed only coronal alignment and accept that sagittal and rotational malalignment may influence the revision rate. This can be the basis for further studies.

Our medium- to early long-term survival of total knee replacement does not seem to be significantly influenced by the immediate post-operative coronal alignment. This may be attributable to modern implants, which may be able to accommodate and withstand slight variations in the limb alignment. However, longer follow-up is required to confirm or repudiate our findings. If indeed longer followup confirms that post-operative alignment influences the survival and eventual outcome then the need for investment into various image and computer-guided systems to achieve a set tibiofemoral alignment can be investigated. Our results showed no significant association between post-operative radiographic coronal knee alignment and revision surgery in the groups studied at a median follow-up of 10 years. Therefore, postoperative alignment on its own does not appear to influence the outcome. We support the conclusion by Smith et al. [11] and Tew et al. [10] that alignment may not be as important a cause of failure as has been previously thought.

\section{References}

1. Bargren JH, Blaha JD, Freeman MAR (1983) Alignment in total knee arthroplasty. Clin Orthop Relat Res 173:178-183

2. Freeman MAR, Todd RC, Bambert $P$ et al (1978) ICLH arthroplsty of the knee: 1968-1977. J Bone Joint Surg 60-B: 339-344

3. Harvey IA, Manning MP, Sampath SAC et al (1995) Alignment of total knee arthroplasty: the relationship to radiolucency around the tibial component. Med Eng Phys 17:182-187

4. Hood RW, Vanni M, Insall JN (1981) The correction of knee alignment in 225 consecutive total condylar knee replacement. Clin Orthop 160:94-105

5. Insall JN, Binazzi R, Soudry $M$ et al (1985) Total knee arthroplasty. Clin Orthop Relat Res 192:13-22

6. Insall JN (1984) Total knee replacement. In: Insall JN (ed) Surgery of the knee. Churchill Livingstone, New York

7. Jeffery RS, Morris RW, Denham RA (1991) Coronal alignment after total knee replacement. J Bone Joint Surg 73 B:709-714

8. Knutson K, Lindstrand A, Lindgren L (1986) Survival of knee arthroplasties: a nationwide multicentre investigation of 8000 cases. J Bone Joint Surg 68-B:795-803

9. Reckling FW, Asher MA, Dillon WL (1977) A longitudinal study of radiolucent line at the bone-cement following total joint replacement procedures. J Bone Joint Surg 59-A:355-358

10. Ritter MA, Faris PM, Keating EM et al (1994) Postoperative alignment of total knee replacement: its effect on survival. Clin Orthop Relat Res 299:153-156

11. Smith JL Jr, Tullos HS, Davidson JP (1989) Alignment of total knee arthroplasty. J Arhtroplasty 4 Suppl S:55-61

12. Tew M, Waugh W (1985) Tibiofemoral alignment and the results of knee replacement. J Bone Joint Surge 67-B:551-556

13. Petersen TL, Engh GA (1988) Radiographic Assessment of Knee Alignment after total knee arthroplasty. J Arthroplasty 31:67-72 